Artículo

\title{
Ecuación determinística para el diseño hidráulico de sistemas de riego por salidas múltiples
}

\author{
José Guadalupe González-Quirino ${ }^{1}$ \\ Manuel Fortis-Hernández ${ }^{1}$ \\ Pablo Preciado-Rangel ${ }^{1}$ \\ Pablo Yescas-Coronado ${ }^{1}$ \\ Juan Manuel Barrios-Díaz ${ }^{3}$ \\ Arturo Reyes-González ${ }^{2 \S}$ \\ ${ }^{1}$ Tecnológico Nacional de México-Campus Instituto Tecnológico de Torreón (TecNm-ITT). Antigua carretera \\ Torreón-San Pedro km 7.5, Torreón, Coahuila, México. CP. 27170. Tel. 8717507199. \\ (gonzalezquirino@hotmail.co; pyescas@hotmail.com; textrc2010@live.com; ppreciador@yahoo.com.mx). \\ ${ }^{2}$ INIFAP. Boulevard José Santos Valdez 1200, Matamoros, Coahuila. (reyes.arturo@inifap.gob.mx). \\ ${ }^{3}$ Benemérita Universidad Autónoma de Puebla-Facultad en Ingeniería Agrohidráulica. Avenida Universidad \\ s/n, San Juan Acateno, Teziutlan, Puebla. CP. 73965. Tel. 231 3122933. (juan.barrios@ correo.buap.mx). \\ ${ }^{\S}$ Autor para correspondencia: reyes.arturo@inifap.gob.mx.
}

\section{Resumen}

En el diseño de sistemas de riego de multisalidas es necesario calcular las pérdidas de energía por fricción en tuberías y determinar el diámetro más económico y óptimo, para ello existen las ecuaciones de Manning, Hanzen \& Williams y Darcy \& Wesbach. De acuerdo con lo anterior el objetivo de este trabajo fue realizar un análisis físico matemático a las ecuaciones de pérdidas de energía por rozamiento en tuberías y accesorios para generar al menos una ecuación determinística basada en la ecuación de Manning y Darcy \& Wesbach para el diseño hidráulico del riego por multisalidas y reducir altos costos en la operación de los equipos de riego agrícola. El estudio se llevó a cabo en primavera de 2021, en el Tecnológico Nacional de México, Campus Instituto Tecnológico de Torreón. Se fusionaron algebraicamente las ecuaciones de pérdidas de energía localizada y por rozamiento (Darcy \& Wesbach y Manning) y se obtuvieron tres ecuaciones determinísticas para el diseño de salidas múltiples con un rango de error relativo de -0.009 a 0.209\% con relación a la ecuación propuesta por la SARH 1979 (CENAMAR).

Palabras clave: Darcy \& Wesbach, ecuación determinística, Manning, pérdidas de energía.

Recibido: abril de 2021

Aceptado: julio de 2021 


\section{Introducción}

En el diseño de sistemas de riego de multisalidas existe la necesidad de calcular las pérdidas de energía por fricción en tuberías (Pimenta et al., 2018) y determinar el diámetro más económico y óptimo (Espinosa et al., 2016; Melo et al., 2019). Está pérdida se debe a la fricción entre las partículas del fluido y la pared de la tubería (Nosrati et al., 2017), para ello existen las ecuaciones de Manning, Hanzen \& Williams y Darcy \& Wesbach. Estas ecuaciones poseen un coeficiente de salidas múltiples o un factor de salidas o de fricción que dificulta el cálculo del número de emisores goteros (emisores).

Además, la ecuación de Hanzen \& Williams sobreestima los costos de pérdidas de energía por fricción en $13 \%$ en comparación con Darcy \& Wesbach (Flecha et al., 2010), lo cual encarece la operación de los equipos de riego. Alegret y Martínez (2019) propusieron un coeficiente a la ecuación de Hazen \& Williams basado en la rugosidad relativa y el número de Reynolds, obteniendo valores muy semejantes a las pérdidas de energía de la ecuación de Darcy \& Wesbach. Adams (2016) combinó la rugosidad de granos de arena con la ecuación de Darcy \& Wesbach y la ecuación Coolebrock \& White y determinó el coeficiente de rugosidad de Hazen \& Williams con una desviación de $2.3 \%$.

Jamil y Mujeebu (2019); Jamil (2019) determinaron una ecuación para calcular las pérdidas de energía con Darcy \& Wesbach sin el uso del factor de fricción y número de Reynolds. Jiménez y Ramírez (2018) combinaron la ecuación de Manning y Darcy \& Wesbach con el factor de salidas múltiples de Christiansen para determinar el número de salidas a través del método numérico de Newton Raphson. Wang et al. (2016) realizaron un análisis hidráulico de una unidad de riego por goteo con elementos finitos y un método iterativo de eliminación gaussiana y determinó que una línea regante por multisalidas es una alternativa a un sistema ramificado. Hassan (2017), desarrolló un modelo matemático de programación lineal en el que evaluó los costos y pérdidas de energía en diferentes diámetros de tuberías y determinó que las mejores ecuaciones para pérdidas de energía fueron las de Darcy \& Wesbach y Manning. Se realizó un análisis físico matemático a las ecuaciones de pérdidas de energía por rozamiento en tuberías y accesorios para generar al menos una ecuación determinística basada en la ecuación de Manning y Darcy \& Wesbach para el diseño hidráulico del riego por multisalidas.

\section{Materiales y métodos}

El presente trabajo se llevó a cabo en las instalaciones del Instituto Tecnológico de Torreón, localizado en el ejido Ana, carretera Torreón San Pedro kilómetro 7.5, en los paralelos 25 36' 53', de latitud norte y $103^{\circ} 22^{\prime} 21^{\prime}$ ' de longitud oeste.

\section{Ecuación de pérdida de energía}

Hassan (2017), desarrolló un modelo matemático de programación lineal en el que evaluó los costos y pérdidas de energía en cinco diámetros nominales de tuberías 3", 3-1/2", 4", 5" y 6" con las ecuaciones de Hazen-Williams, Manning, Scobey y Darcy \& Weisbach y determinó que las mejores ecuaciones para pérdidas de energía fueron las de Darcy \& Wesbach y Manning. Por tal motivo en este trabajo se utilizaron las ecuaciones de Manning y Darcy \& Wesbach. 


\section{Ecuación de pérdida de energía por rozamiento}

A continuación, se presenta la ecuación 1 de pérdidas de energía por fricción de Manning. Hf= $\frac{4^{\frac{10}{3}} \mathrm{n}^{2} \mathrm{Lq}^{2}}{\pi^{2} \mathrm{~d}^{\frac{16}{3}}}$

1). Donde: $\mathrm{Hf}=$ pérdida de energía por fricción $(\mathrm{m}) ; \mathrm{q}=$ caudal de la tubería $\left(\mathrm{m}^{3} \mathrm{~s}^{-1}\right) ; \mathrm{L}=$ longitud de la tubería (m); $\mathrm{n}=$ coeficiente de rugosidad de Manning; y d= diámetro de la tubería (m).

\section{Ecuación de pérdida de energía localizada}

La ecuación de pérdidas de energía localizada de Darcy \& Wesbach. $H L=K_{L} \frac{v^{2}}{2 g}$ 2). Donde: HL= pérdida de energía localizada $(\mathrm{m}) ; \mathrm{K}_{\mathrm{L}}=$ coeficiente de pérdida de energía localizada; $\mathrm{v}=$ velocidad del fluido (m); y g= aceleración de la gravedad $\left(\mathrm{m} \mathrm{s}^{-2}\right)$. Si se sustituye en la ecuación anterior la velocidad en función del caudal y área resulta: $\left.\mathrm{HL}=\mathrm{K}_{\mathrm{L}} \frac{8(\mathrm{q})^{2}}{\mathrm{~g} \pi^{2} \mathrm{~d}^{4}} \quad 3\right)$.

\section{Análisis de una línea regante}

En la Figura 1, se presenta una línea regante de emisores. Al analizar la línea regante de derecha a izquierda, en el tramo de tubería uno circula un caudal ' $q$ ', en el tramo número 2 circula un caudal '2q', en el tramo número 3 circula un caudal '3q', en el tramo número 4 circula un caudal '4q' y así sucesivamente. Esto significa que el caudal aumenta un 'q' en cada tramo de tubería hasta llegar al gasto máximo de la tubería ' $\mathrm{Nq}$ ' en el tramo 'NL'. Por lo tanto, las pérdidas de energía por fricción y localizada se están acumulando en un 'q' en cada tramo de tubería por donde circula el agua hasta tener las pérdidas de energía total en el tramo 'NL' y caudal máximo 'Nq'.

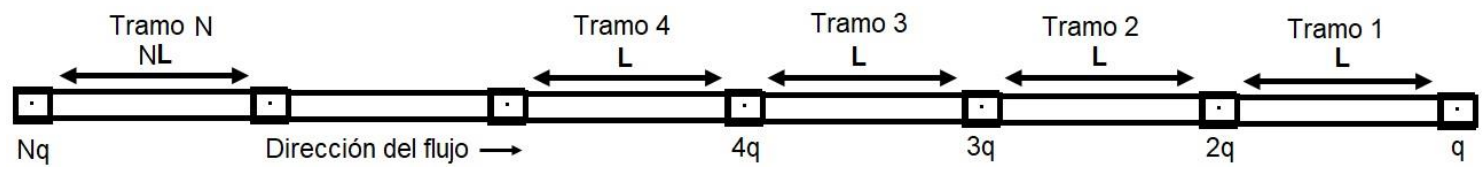

Figura 1. Diagrama de una línea regante de goteros.

Al sumar las pérdidas de energía por rozamiento en cada tramo de derecha a izquierda, usando la ecuación 1, resulta la ecuación (4). $\mathrm{Hf}_{\mathrm{T}}=\frac{4^{\frac{10}{3}} \mathrm{n}^{2} \mathrm{~L}(1 \mathrm{q})^{2}}{\pi^{2} \mathrm{~d}^{\frac{16}{3}}}+\frac{4^{\frac{10}{3}} \mathrm{n}^{2} \mathrm{~L}(2 \mathrm{q})^{2}}{\pi^{2} \mathrm{~d}^{\frac{16}{3}}}+\frac{4^{\frac{10}{3}} \mathrm{n}^{2} \mathrm{~L}(3 \mathrm{q})^{2}}{\pi^{2} \mathrm{~d}^{\frac{16}{3}}} \ldots+\frac{4^{\frac{10}{3}} \mathrm{n}^{2} \mathrm{~L}(\mathrm{Nq})^{2}}{\pi^{2} \mathrm{~d}^{\frac{16}{3}}}=$ $\frac{4^{\frac{10}{3}} \mathrm{n}^{2} \mathrm{~L}(\mathrm{q})^{2}}{\pi^{2} \mathrm{~d}^{\frac{16}{3}}} \sum_{\mathrm{i}=1}^{\mathrm{N}} \mathrm{i}^{2}$ 4). Al sumar las pérdidas de energía localizada en cada emisor de derecha a izquierda, con la ecuación (3) resulta la ecuación (5). $\mathrm{HL}_{\mathrm{T}}=$ $\mathrm{K}_{\mathrm{L}} \frac{8(1 \mathrm{q})^{2}}{\mathrm{~g} \pi^{2} \mathrm{~d}^{4}}+\mathrm{K}_{\mathrm{L}} \frac{8(2 \mathrm{q})^{2}}{\mathrm{~g} \pi^{2} \mathrm{~d}^{4}}+\mathrm{K}_{\mathrm{L}} \frac{8(3 \mathrm{q})^{2}}{\mathrm{~g} \pi^{2} \mathrm{~d}^{4}}+\mathrm{K}_{\mathrm{L}} \frac{8(4 \mathrm{q})^{2}}{\mathrm{~g} \pi^{2} \mathrm{~d}^{4}}+\ldots \mathrm{K}_{\mathrm{L}} \frac{8(\mathrm{Nq})^{2}}{\mathrm{~g} \pi^{2} \mathrm{~d}^{4}}=\mathrm{K}_{\mathrm{L}} \frac{8(\mathrm{q})^{2}}{\mathrm{~g} \pi^{2} \mathrm{~d}^{4}} \sum_{\mathrm{i}=1}^{\mathrm{N}} \mathrm{i}^{2}$ 5). Al sumar las pérdidas de energía total de rozamiento ' $\mathrm{Hf}_{\mathrm{T}}$ ' (4), total localizada $\mathrm{HL}_{\mathrm{T}}(5)$ y si se factoriza, resulta la ecuación de pérdidas de energía total en un línea regante (6). $\left.\mathrm{Hf}_{\mathrm{TLR}}=\left(\frac{4^{\frac{10}{3} \mathrm{n}^{2} \mathrm{Lq}^{2}}}{\pi^{2} \mathrm{~d}^{\frac{16}{3}}}+\mathrm{K}_{\mathrm{L}} \frac{8 \mathrm{q}^{2}}{\mathrm{g \pi ^{2 } \mathrm { d } ^ { 4 }}}\right) \sum_{\mathrm{i}=1}^{\mathrm{N}} \mathrm{i}^{2} 6\right)$. 
Solucionando la sumatoria de la ecuación (6) y sustituyéndola en la misma ecuación, resulta la ecuación (7). $\left.\left(\frac{4^{\frac{10}{3}} \mathrm{n}^{2} \mathrm{Lq}^{2}}{\pi^{2} \mathrm{~d}^{\frac{16}{3}}}+\mathrm{K}_{\mathrm{L}} \frac{8 \mathrm{q}^{2}}{\mathrm{~g}^{2} \mathrm{~d}^{4}}\right)\left(\frac{2 \mathrm{~N}^{3}+3 \mathrm{~N}^{2}+\mathrm{N}}{6}\right)=\mathrm{Hf}_{\text {Total }} 7\right)$. Acomodando términos " $\mathrm{N}$ " de la ecuación anterior resulta un polinomio de energía total de la línea regante (8). $2 \mathrm{~N}^{3}+3 \mathrm{~N}^{2}+\mathrm{N}$ $\frac{6 \mathrm{Hf}_{\text {Total }}}{\left(\frac{4^{\frac{10}{3}} \mathrm{n}^{2} \mathrm{Lq}^{2}}{\pi^{2} \mathrm{~d}^{\frac{16}{3}}}+\mathrm{K}_{\mathrm{L}} \frac{8 \mathrm{q}^{2}}{\mathrm{~g} \pi^{2} \mathrm{~d}^{4}}\right)}=0$

\section{Solución del polinomio de energía de la línea regante}

Se extrae el termino constante de la ecuación (8) y si se analiza algebraicamente resulta la ecuación (8.1). $\mathrm{K}=\frac{3 / 4 * \mathrm{Hf}_{\text {Total }} * \pi^{2} \mathrm{gd}^{\frac{16}{3}}}{4^{\frac{11}{6}} \mathrm{gn}^{2} \mathrm{sq}^{2}+\mathrm{K}_{\mathrm{L}} \mathrm{q}^{2} \mathrm{~d}^{\frac{4}{3}}}$ 8.1). Al compactar la ecuación (8) se transforma en la ecuación (9). $\mathrm{F}(\mathrm{N})=2 \mathrm{~N}^{3}+3 \mathrm{~N}^{2}+\mathrm{N}-\mathrm{K}=0$ 9). La ecuación anterior está formada por los siguientes coeficientes; $\mathrm{a}=$ $2, \mathrm{~b}=3, \mathrm{c}=1 \mathrm{y} \mathrm{d}=-\mathrm{K}$.

A continuación, se presenta la ecuación (10) que soluciona la ecuación (9) al generar la solución

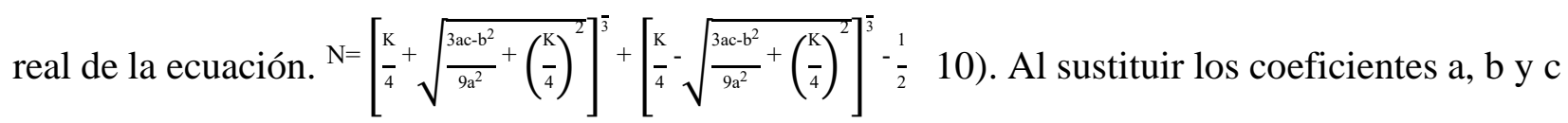
de la ecuación (9) en la ecuación (10) se obtiene la ecuación (11) que es la solución de la ecuación

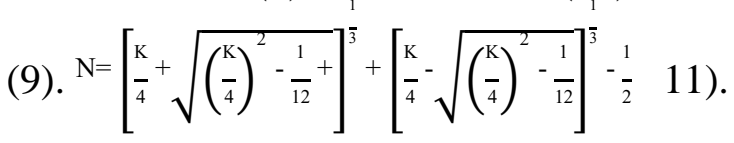

\section{Análisis de la solución real del polinomio de energía total de la línea regante}

La ecuación 11 está integrada por tres términos $\left(1^{\circ}, 2^{\circ}\right.$ y $\left.3^{\circ}\right)$, los dos primeros términos contienen una variable y el tercer término es una constante, dichos términos se presentan a continuación (ecuaciones $11.1,11.2$ y 11.3, respectivamente). $\left.1^{e r}=\left[\frac{\mathrm{K}}{4}+\sqrt{\left(\frac{\mathrm{K}}{4}\right)^{2}-\frac{1}{12}}\right]^{\frac{1}{3}} 11.1\right) ; 2^{\mathrm{do}}=\left[\frac{\mathrm{K}}{4}-\sqrt{\left(\frac{\mathrm{K}}{4}\right)^{2}-\frac{1}{12}}\right]^{\frac{1}{3}}$ $\left.11.2) ; 3^{e r}=-\frac{1}{2} \quad 11.3\right)$.

\section{Análisis estadístico del número de salidas}

Se determinó el error relativo porcentual, para ello se adaptó la ecuación de Olivares et al. (2019), quedando la siguiente ecuación. Error relativo $(\%)=\left(\frac{N e-N}{N}\right) 100$ 12). Donde: error relativo $(\%)=$ es el por ciento de error relativo, $\mathrm{N}=$ número de emisores con la ecuación (11); $\mathrm{Ne}=$ número de goteros estimado con la ecuación 11.1.

Para estimar este error se utilizó el apoyo de una hoja de cálculo electrónica (Excel) para cada una de las ecuaciones que determinan el número de salidas. En el Cuadro 1 se observa el valor numérico de ' $\mathrm{N}$ ' número de emisores (11), el error en el porcentaje de ' $\mathrm{N}$ ' número de emisores con relación al primer término ' $1{ }^{\mathrm{er}}$ ' $(11.1)$, la solución parcial de los términos $\left(1^{\circ}, 2^{\circ}\right.$ y $\left.3^{\circ}\right)$ a la solución total del polinomio, así como la aportación a la solución en porcentaje de cada uno de los términos. 
En este cuadro se aprecia que la aportación del primer término ' $11^{\circ}$ ' en la columna de soluciones parciales la parte decimal de estos valores tienden a ser constante a medida que $\mathrm{N}$ aumenta y en la columna de aportación a la solución tiende a disminuir. La aportación del segundo término ' $2^{\circ}$ ' en la columna de soluciones parciales tiende a cero cuando $\mathrm{N}$ ' toma valores iguales o mayores de 87 y en la columna de aportación a la solución tiende a un valor pequeño cuando ' $N$ ' es igual a 87 salidas. Asimismo, la aportación del tercer término ' $3^{\circ}$ ' sirve como factor de corrección a la solución y tiende a disminuir significativamente en el mismo número de salidas en la columna de aportación a la solución. Esto significa que la solución compacta de la ecuación (9) se resume en el primer ' $1{ }^{\circ}$ y tercer ' $3{ }^{\circ}$ ' término de la ecuación (11), dando como resultado la ecuación 13 . $\mathrm{N}=$ $\left.\left[\frac{\mathrm{K}}{4}+\sqrt{\left(\frac{\mathrm{K}}{4}\right)^{2}-\frac{1}{12}}\right]^{\frac{1}{3}}-\frac{1}{2} \quad 13\right)$.

Cuadro 1. Análisis de la solución real del polinomio de energía total de la línea regante.

\begin{tabular}{|c|c|c|c|c|c|c|c|c|c|}
\hline \multirow{2}{*}{ K } & \multirow{2}{*}{$\mathrm{N}$} & \multirow{2}{*}{ Error 'N' (\%) } & \multicolumn{3}{|c|}{ Soluciones parciales } & \multicolumn{4}{|c|}{ Aportación a la solución (\%) } \\
\hline & & & $1^{\circ}$ & $2^{\circ}$ & $3^{\circ}$ & $1^{\circ}$ & $2^{\circ}$ & $3^{\circ}$ & $1^{\circ}+3^{\circ}$ \\
\hline 2.8 & 1 & 10.38 & 1.1 & 0.4 & -0.5 & 110.38 & 39.71 & -50.09 & 60.29 \\
\hline 25 & 2 & 15.52 & 2.32 & 0.19 & -0.5 & 115.52 & 9.37 & -24.89 & 90.63 \\
\hline 77 & 3 & 12.33 & 3.38 & 0.13 & -0.5 & 112.33 & 4.30 & -16.63 & 95.7 \\
\hline 171 & 4 & 10.01 & 4.41 & 0.1 & -0.5 & 110.01 & 2.48 & -12.49 & 97.52 \\
\hline 319 & 5 & 8.38 & 5.42 & 0.08 & -0.5 & 108.38 & 1.61 & -9.99 & 98.39 \\
\hline 533 & 6 & 7.2 & 6.44 & 0.07 & -0.5 & 107.2 & 1.13 & -8.33 & 98.87 \\
\hline 825 & 7 & 6.3 & 7.44 & 0.06 & -0.5 & 106.3 & 0.84 & -7.14 & 99.16 \\
\hline 1206 & 8 & 5.6 & 8.45 & 0.05 & -0.5 & 105.6 & 0.65 & -6.25 & 99.35 \\
\hline 2288 & 10 & 4.58 & 10.46 & 0.04 & -0.5 & 104.58 & 0.42 & -5 & 99.58 \\
\hline 10673 & 17 & 2.79 & 17.48 & 0.02 & -0.5 & 102.79 & 0.15 & -2.94 & 99.85 \\
\hline 51268 & 29 & 1.67 & 29.49 & 0.01 & -0.5 & 101.67 & 0.05 & -1.72 & 99.95 \\
\hline 257445 & 50 & 0.98 & 50.49 & 0.01 & -0.5 & 100.98 & 0.02 & -1 & 99.98 \\
\hline 1339615 & 87 & 0.57 & 87.5 & 0 & -0.5 & 100.57 & 0.01 & -0.57 & 99.99 \\
\hline 458199 & 133 & 0.37 & 133.5 & 0 & -0.5 & 100.37 & 0 & -0.38 & 100 \\
\hline 20015200 & 215 & 0.23 & 215.5 & 0 & -0.5 & 100.23 & 0 & -0.23 & 100 \\
\hline 78955400 & 340 & 0.15 & 340.5 & 0 & -0.5 & 100.15 & 0 & -0.15 & 100 \\
\hline 576300000 & 660 & 0.08 & 660.5 & 0 & -0.5 & 100.08 & 0 & -0.08 & 100 \\
\hline 2003002000 & 1000 & 0.05 & 1000.5 & 0 & -0.5 & 100.05 & 0 & -0.05 & 100 \\
\hline
\end{tabular}

Datos calculados con las ecuaciones $11.1,11.2,11.3,12$ y 13. Donde: $\mathrm{K}=$ constante del polinomio; $\mathrm{N}=$ número de emisores; $1^{\circ}=$ primer término; $2^{\circ}=$ segundo término; y $3^{\circ}=$ tercer término de la ecuación.

La ecuación 13 está formada por dos subtérminos variables, los cuales se presentan a continuación. Subtérmino $1=\frac{K}{4} \quad 13.1$ ); Subtérmino $\left.2=\sqrt{\left(\frac{K}{4}\right)^{2}-\frac{1}{12}} \quad 13.2\right)$. 
En el Cuadro 2 se observa el valor numérico de ' $\mathrm{N}$ ' número de emisores (11), los valores del 'subtérmino 1' (13.1) y 'subtérmino 2' (13.2), el valor numérico de 'N modificada', calculada con la suma de las ecuaciones 13.1 y 13.2 que representa la ecuación (11.1); el 'error de N' determinada con los valores de 'N modificada' y los valores de 'N', calculado con la ecuación (12).

En este cuadro se observa la aportación del subtérmino 1 y subtérmino 2, en donde se observa que existe poca diferencia entre las dos columnas cuando ' $\mathrm{N}$ ' toma valores de uno a siete y los valores de los dos subtérminos son iguales cuando $\mathrm{N}$ es igual o mayor a ocho. Esto es debido a que la resta de un doceavo al segundo término de la ecuación no afecta al valor del subtérmino dos, al comparar la aportación de los dos subtérminos cuando ' $\mathrm{N}$ ' toma un valor de 87 el 'error de N' calculado con la ecuación 12 es de 0.6 por ciento. Por lo tanto, se elimina el valor de $-1 / 12$ del 'subtérmino 2', dando origen a la ecuación (13.21). Subtérmino $\left.2=\frac{K}{4} 13.21\right)$.

Cuadro 2. Análisis de los subtérminos de la ecuación 21 (solución real del polinomio de energía total de la línea regante).

\begin{tabular}{cccccc}
\hline K & N & Subtérmino 1 & Subtérmino 2 & N modificada & Error de 'N' (\%) \\
\hline 2.9 & 1 & 0.7 & 0.7 & 1.1 & 10.8 \\
25 & 2 & 6.3 & 6.2 & 2.3 & 15.5 \\
77 & 3 & 19.3 & 19.2 & 3.4 & 12.3 \\
171 & 4 & 42.8 & 42.7 & 4.4 & 10 \\
319 & 5 & 79.8 & 79.7 & 5.4 & 8.4 \\
533 & 6 & 133.3 & 133.2 & 6.4 & 7.2 \\
825 & 7 & 206.3 & 206.2 & 7.4 & 6.3 \\
1206 & 8 & 301.5 & 301.5 & 8.4 & 5.6 \\
2288 & 10 & 572 & 572.0 & 10.5 & 4.6 \\
4886 & 13 & 1221.5 & 1221.5 & 13.5 & 3.6 \\
10673 & 17 & 2668.3 & 2668.2 & 17.5 & 2.8 \\
51268 & 29 & 12817 & 12817 & 29.5 & 1.7 \\
257445 & 50 & 64361.3 & 64361.2 & 50.5 & 1 \\
1339615 & 87 & 334903.8 & 334.903 .7 & 87.5 & 0.6 \\
4758199 & 133 & 1189549.8 & 1189549.7 & 133.5 & 0.4 \\
11761000 & 180 & 2940250 & 2940250 & 180.5 & 0.3 \\
20015200 & 215 & 5003800 & 5003800 & 215.5 & 0.2 \\
78955400 & 340 & 19738850 & 19738850 & 340.5 & 0.1 \\
576300000 & 660 & 144075000 & 144075000 & 660.5 & 0.1 \\
2003002000 & 1000 & 500750500 & 500750500 & 1000.5 & 0 \\
\hline
\end{tabular}

Datos calculados con las ecuaciones 11, 13.1, 13.2 y 12 .

Al fusionar el subtérmino 1 (13.1) con el subtérmino 2 (13.21) resulta la ecuación (14) que se presenta a continuación: $N=\left[\frac{\mathrm{K}}{2}\right]^{\frac{1}{3}} 14$ ). 


\section{Ecuaciones determinísticas}

Al unir la ecuación (8.1) con la ecuación (14) resulta la ecuación (15). $\left.\mathrm{N}=\left[\frac{3 / 8\left(\mathrm{Hf}_{\mathrm{Total}}\right) \pi^{2} \mathrm{~g} \mathrm{~d}^{\frac{16}{3}}}{4^{\frac{11}{3}} \mathrm{gn}^{2} \mathrm{sq}^{2}+\mathrm{K}_{\mathrm{L}} \mathrm{q}^{2} \mathrm{~d}^{\frac{4}{3}}}\right]^{\frac{1}{3}} 15\right)$. A la ecuación (15) al agregarle el término constante (11.3) el cual posee un valor de (-1/2) resulta la ecuación (16). $\left.\mathrm{N}=\left[\frac{3 / 8\left(\mathrm{Hf}_{\mathrm{Total}}\right) \pi^{2} \mathrm{~g} \mathrm{~d}^{\frac{16}{3}}}{4^{\frac{11}{3}} \mathrm{gn}^{2} \mathrm{sq}^{2}+\mathrm{K}_{\mathrm{L}} \mathrm{q}^{2} \mathrm{~d}^{\frac{4}{3}}}\right]^{\frac{1}{3}}-\frac{1}{2} 16\right)$.

En el Cuadro 3 se observan los 'valores de N' calculados con las ecuaciones (15) y (16) y sus respectivos errores relativos tomando como base la ecuación (11), calculados con la ecuación (12). La ecuación (16) a medida que $\mathrm{N}$ es mayor de 87 emisores los errores son cero en comparación con la ecuación (11), mientras que en la ecuación (15) los errores tienden a cero a partir de los 87 emisores. La ecuación (16) es la que tiene un mejor ajuste que la ecuación (15) con errores que van del $-37.28 \%$ para un emisor y del $-0.01 \%$ para 87 emisores. La ecuación (15) tiene un menor ajuste en comparación con la ecuación (11) con errores que van del $12.35 \%$ para un emisor y del $0.56 \%$ para 87 emisores.

Cuadro 3. Errores relativos de las ecuaciones 15 y 16, con relación a la ecuación 11.

\begin{tabular}{|c|c|c|c|c|c|}
\hline \multirow{2}{*}{$\mathrm{K}$} & \multicolumn{3}{|c|}{ Valores de ' $N$ ' en las ecuaciones } & \multicolumn{2}{|c|}{ Errores de 'N' $(\%)$} \\
\hline & 11 & 15 & 16 & 15 & 16 \\
\hline 2.9 & 1 & 1.1 & 0.6 & 12.35 & -37.28 \\
\hline 25 & 2 & 2.3 & 1.8 & 15.54 & -9.35 \\
\hline 171 & 4 & 4.4 & 3.9 & 10.01 & -2.48 \\
\hline 533 & 6 & 6.4 & 5.9 & 7.2 & -1.13 \\
\hline 1206 & 8 & 8.4 & 7.9 & 5.6 & -0.65 \\
\hline 2288 & 10 & 10.5 & 10 & 4.58 & -0.42 \\
\hline 10673 & 17 & 17.5 & 17 & 2.79 & -0.15 \\
\hline 51268 & 29 & 29.5 & 29 & 1.67 & -0.05 \\
\hline 257445 & 50 & 50.5 & 50 & 0.98 & -0.02 \\
\hline 1339615 & 87 & 87.5 & 88.3 & 0.56 & -0.01 \\
\hline 11761000 & 180 & 180.5 & 180 & 0.28 & 0 \\
\hline 78955400 & 340 & 340.5 & 340 & 0.15 & 0 \\
\hline 576300000 & 660 & 660.5 & 660 & 0.08 & 0 \\
\hline 2003002000 & 1000 & 1000.5 & 1000 & 0.05 & 0 \\
\hline
\end{tabular}

Datos calculados con las ecuaciones $11,12,15$ y 16.

\section{Validación de las ecuaciones determinísticas}

Para la validación de las ecuaciones determinísticas (11, 15 y 16) fue necesario diseñar una línea regante en una hoja de cálculo Excel. Para ello se presentan los siguientes datos y cálculos. 


\section{Diseño de una línea regante}

Se diseñó una línea regante con la metodología propuesta por el Centro Nacional de Métodos Avanzados de Riego (CANAMAR, 1979) perteneciente a la Secretaría de Agricultura y Recursos Hidráulicos (SARH).

\section{Selección del gotero}

Se seleccionó un gotero no auto compensado con un gasto mayor a un litro por hora, el gotero seleccionado fue el de bajo gasto Rivulis E1000 (Rivulis Irrigation Inc; San Diego, CA, EUA), los datos técnicos se presentan en el Cuadro 4.

Cuadro 4. Comportamiento hidráulico del gotero de bajo gasto Rivulis E1000.

\begin{tabular}{cc}
\hline Carga hidráulica $(\mathrm{m})$ & Caudal $\left(\mathrm{L} \mathrm{h}^{-1}\right)$ \\
\hline 8.156 & 1.84 \\
10.195 & 2.05 \\
12.234 & 2.25 \\
14.273 & 2.42 \\
16.312 & 2.594 \\
18.351 & 2.74 \\
20.39 & 2.864 \\
\hline
\end{tabular}

Datos técnicos Rivulis E1000.

\section{Modelo hidráulico del gotero}

Los datos técnicos del gotero se sometieron a un análisis de regresión potencial en una hoja de Excel, los resultados se presentan en la Figura 2.

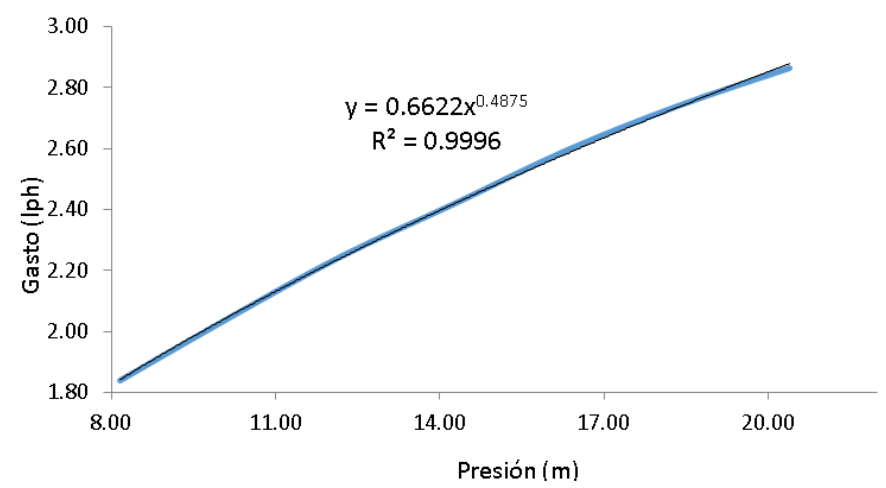

Figura 2. Análisis de regresión del gotero (Rivulis E1000) para estimar los valores de $\mathbf{k}$ y x.

Los resultados permitieron determinar los componentes del modelo hidráulico de goteros, donde el valor de ' $k$ ' coeficiente fue igual a 0.6622 y el valor de ' $x$ ' de 0.4875 exponente (Figura 2). 


\section{Cálculo de la presión de operación de la línea regante}

También se determinó la presión de operación de la línea regante de goteros con la ecuación (17). $\mathrm{H}_{0}=\left[\frac{\mathrm{qm}}{\mathrm{k}\left(1-\frac{\% \mathrm{Vg}}{100}\right)^{0.5}}\right]^{\frac{1}{\mathrm{x}}}$ 17). Donde: $\mathrm{Ho}=$ carga hidráulica de operación del gotero $(\mathrm{m}) ; \mathrm{qm}=$ caudal medio del gotero $\left(2 \mathrm{~L} \mathrm{~h}^{-1}\right), \% \mathrm{Vg}=$ por ciento de variación de gasto entre en primer y el último gotero $(5 \%)$.

\section{Cálculo de pérdida de energía permisible en la línea regante}

El cálculo de la pérdida de energía permisible se determinó con la ecuación (18). Dh= $\mathrm{H}_{\mathrm{o}}[1$ $\left.\left(\frac{100-\% \mathrm{Vg}}{100}\right)^{\frac{1}{x}}\right]$ 18). Donde: $\mathrm{Dh}=$ pérdida de energía permisible $(\mathrm{m})$.

\section{Selección de diámetros para el cálculo del número de goteros en la línea regante}

Se seleccionaron ocho diámetros de tubería de PVC (policloruro de vinilo) para ello se seleccionó la serie inglesa Amanco (Orbia Inc; San Francisco CA, EUA) debido a que este tipo de tubería posee diámetros internos semi equidistantes (Cuadro 5).

Cuadro 5. Diámetros internos de tubería de PVC serie inglesa Amanco.

\begin{tabular}{ccccc}
\hline \multirow{2}{*}{ Diámetro nominal $(\mathrm{mm})$} & \multicolumn{4}{c}{ Diámetros internos de tuberías (mm) } \\
\cline { 2 - 5 } 13 & RD-13.5 & RD-26 & RD-32.5 & RD-41 \\
25 & 17.5 & & \\
38 & 27.8 & 29.8 & \\
50 & 40.5 & 43.9 & & \\
75 & 50.7 & 55.1 & & \\
100 & & 81.5 & & 108.1 \\
150 & & 104.9 & 106.7 & 159.5 \\
200 & & 154.5 & 157.3 & 207.9 \\
\hline
\end{tabular}

Amanco (San Francisco, USA).

\section{Cálculo del número de goteros en diferentes diámetros internos de tubería}

Se diseñó una línea regante determinando el número de goteros se pueden instalar en diferentes

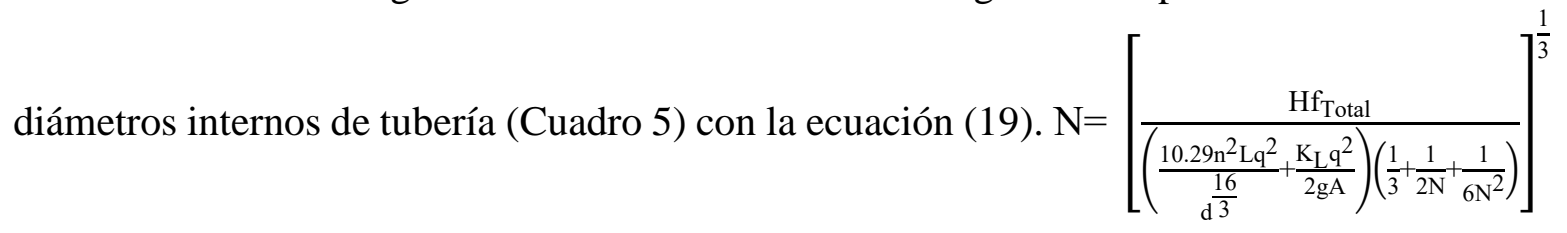
19). 
Donde: $\mathrm{N}=$ número de goteros; $\mathrm{A}=$ área transversal de la tubería $\left(\mathrm{m}^{2}\right) ; \mathrm{K}_{\mathrm{L}}=$ coeficiente de inserción del gotero (0.5); $\mathrm{n}=$ coeficiente de rugosidad de la tubería de PVC (0.0079); $\mathrm{Hf}_{\text {Total }}=$ pérdida de energía total, se diseñó una sola línea regante para este caso $\mathrm{Hf}_{\text {Total }}=\mathrm{Dh}$. El número de goteros se calculó con la ecuación 19 y con las ecuaciones 11, 15 y 16. El por ciento de error relativo se determinó con la ecuación 12 tomando como base la ecuación 19 (Cuadro 6) (análisis estadístico del número de salidas).

\section{Resultados y discusión}

Al generar las ecuaciones determinísticas para el diseño de salidas múltiples (ecuación 11, 15 y 16) se realizó un análisis integral de pérdidas de energía en tuberías asociadas a la rugosidad, accesorios de acuerdo a Haddad (2019); Tas et al. (2020), y diámetros de acuerdo a Vilaça et al. (2017), en el diseño y análisis hidráulico se tomó en cuenta el caudal de salida del emisor de acuerdo a Sadeghi et al. (2016); asimismo, se determinó con precisión las pérdidas de energía en los accesorios de la línea regante ya que pueden variar de 6.3 a un 49\% (Wang y Chen, 2018). Sin embargo, Wang et al. (2019) reportan que la relación entre la pérdida de carga menor y la total en una tubería puede llegar a un $71.71 \%$.

De acuerdo con las discrepancias de literatura de pérdidas de energía en accesorios o localizadas, se tomó en cuenta la ecuación de pérdida de energía localizada algebraicamente (4) en la generación de las tres ecuaciones determinísticas $(11,15$ y 16). Con lo que respecta a pérdidas de energía por rozamientos en tuberías, Monserrat et al. (2018) señala que la longitud máxima de una línea regante en suelo nivelado tiende a ser igual para cualquier pendiente y facilita la optimización económica, mientras que Wang et al. (2018) reportaron que las pérdidas menores y las de rozamiento están estrechamente relacionadas con el espaciamiento entre goteros, motivo por el cual se utilizó algebraicamente la ecuación de pérdidas de energía por rozamiento (5).

En el Cuadro 6, se muestran los errores relativos en por ciento de las tres ecuaciones determinísticas $(11,15$ y 16) en comparación con la ecuación (19). La ecuación (11) tiene un valor mínimo de $0.009 \%$ y un valor máximo de $0.006 \%$, la ecuación 15 tiene un valor mínimo de $0.007 \%$ y un valor máximo de $0.209 \%$ y la ecuación 16 tiene un valor mínimo de $-0.009 \%$ a un valor máximo de $0.008 \%$. La ecuación con mejor ajuste en orden descendente es la 16,11 y 15 , esto se debe a que las ecuaciones 16 y 11 poseen todos los términos de la solución de la ecuación cúbica (19). Sin embargo, el error general de las tres ecuaciones oscila de -0.009 a $0.209 \%$ (Cuadro 6).

Este rango de errores es menor al determinado por Baiamonte (2015) quien presentó un procedimiento de cuatro ecuaciones implícitas para diseñar líneas regantes de emisores con errores relativos menores al 2\% y Baiamonte (2017), que simplificó un procedimiento analítico para el diseño óptima de líneas regantes de emisores con errores inferiores al 1.9\%. Por otra parte, Monge et al. (2019) determinaron las pérdidas de energía en una tubería de conducción de bambú con multicompuertas usando las ecuaciones de Darcy \&Wesbach, Manning y Hazen\& Williams y determinaron que las ecuaciones Darcy \& Wesbach y Manning estiman mejor las pérdidas de energía para tuberías rugosas. Asimismo, Taş y Ağıralioğlu (2018) calcularon las pérdidas de energía en tuberías y determinaron que las ecuaciones de Darcy \& Wesbach y Manning son las mejores. 
Cuadro 6. Valores de ' $N$ ' número de goteros con diferentes ecuaciones y errores relativos para diferentes diámetros internos de tuberías de PVC.

\begin{tabular}{|c|c|c|c|c|c|c|c|}
\hline \multirow{2}{*}{$\begin{array}{l}\text { Diámetro interno } \\
(\mathrm{mm})\end{array}$} & \multicolumn{4}{|c|}{ Valores de 'N' en las diferentes ecuaciones } & \multicolumn{3}{|c|}{ Errores relativos de 'N' $(\%)$} \\
\hline & 19 & 11 & 15 & 16 & 11 & 15 & 16 \\
\hline 17.5 & 229.12 & 229.1 & 229.6 & 229.1 & -0.009 & 0.209 & -0.009 \\
\hline 27.8 & 460.35 & 460.33 & 460.83 & 460.33 & -0.004 & 0.104 & 0.004 \\
\hline 29.8 & 509.82 & 509.86 & 510.36 & 509.86 & 0.008 & 0.106 & 0.008 \\
\hline 40.5 & 795.11 & 795.09 & 795.59 & 795.09 & -0.003 & 0.06 & -0.003 \\
\hline 43.9 & 892 & 892.04 & 892.54 & 892.04 & 0.004 & 0.061 & 0.004 \\
\hline 50.7 & 1093.85 & 1093.92 & 1094.42 & 1093.92 & 0.006 & 0.052 & 0.006 \\
\hline 55.1 & 1229.82 & 1229.8 & 1230.3 & 1229.8 & -0.002 & 0.039 & -0.002 \\
\hline 81.5 & 2118.86 & 2118.83 & 2119.33 & 2118.83 & -0.001 & 0.022 & -0.001 \\
\hline 104.9 & 2995.35 & 2995.32 & 2995.82 & 2995.32 & -0.001 & 0.016 & -0.001 \\
\hline 106.7 & 3065.76 & 3065.73 & 3066.23 & 3065.73 & -0.001 & 0.015 & -0.001 \\
\hline 108.1 & 3120.79 & 3120.77 & 3121.27 & 3120.77 & -0.001 & 0.015 & -0.001 \\
\hline 154.5 & 5069.87 & 5069.84 & 5070.34 & 5069.84 & -0.001 & 0.009 & -0.001 \\
\hline 157.3 & 5194.59 & 5194.56 & 5195.06 & 5194.56 & -0.001 & 0.009 & -0.001 \\
\hline 159.5 & 5284.14 & 5284.11 & 5284.61 & 5284.11 & -0.001 & 0.009 & -0.001 \\
\hline 201.3 & 7247.19 & 7247.17 & 7247.67 & 7247.17 & 0 & 0.007 & 0 \\
\hline 204.9 & 229.12 & 229.1 & 229.6 & 229.1 & -0.009 & 0.209 & -0.009 \\
\hline 207.9 & 326.98 & 326.97 & 327.47 & 326.97 & -0.003 & 0.15 & -0.003 \\
\hline
\end{tabular}

Datos calculados con las ecuaciones $11,15,16$ y 19.

De acuerdo con los resultados de los autores antes mencionados y los resultados del Cuadro 6, los errores relativos de ' $\mathrm{N}$ ' son aceptables para las ecuaciones 11,15 y 16 y se pueden utilizar en el diseño de riego agrícola por goteo y aspersión.

\section{Conclusiones}

Fue posible generar tres ecuaciones determinísticas basadas en las ecuaciones de Darcy \&Wesbach y Manning, (una de solución total ecuación (11) y dos ecuaciones compactas (15) y (16)) para el diseño de sistemas de riego agrícola por goteo. En las tres ecuaciones generadas se observó que a medida que aumenta el número de emisores (goteros) el error de las ecuaciones tiende a cero. El error relativo para el cálculo del número de emisores en diferentes diámetros internos de tubería de PVC para las tres ecuaciones osciló entre $-0.009 \%$ y $0.209 \%$.

\section{Literatura citada}

Adams, T. M. 2016. Improved method for converting equivalent sand-grain roughness to HazenWilliams coefficient. Proceedings of the $2^{\text {nd }}$ World Congress on Mechanical, Chemical, and Material Engineering (MCM 16). Paper No. HTFF 119. Budapest, Hungary. 1-6 pp. Doi: $10.11159 / \mathrm{htff} 16.119$. 
Alegret-Breña, E. and Martínez-Valdés, Y. 2019. Coeficiente de Hazen-Williams en función del número de Reynolds y la rugosidad relativa. La Habana, Cuba. Ingeniería Hidráulica y Ambiental. 40(3):41-55.

Baiamonte, G. 2015. Simple relationships for the optimal design of paired drip laterals on uniform slopes. Palermo, Italy. J. Irrig. Drain. Eng. 142(2):1-9.

Baiamonte, G. 2017. Advances in designing drip irrigation laterals. Palermo, Italy. Agricultural Water Management. Agric. Water Manag.199(2):157-174.

Espinosa-Espinosa, B.; Flores-Magdaleno, H.; Ascencio-Hernández, R. and Carrillo-Flores, G. 2016. Design of a system of farm hydrant with the Turns and Clement methods: technical and economic análisis. Estado de México, México. Terra Latinoam. 34(4):431-440.

Flechas, R. A. 2010. Efecto del uso de la ecuación de Darcy-Weisbach vs la ecuación de HazenWilliams sobre los costos de diseños optimizados en acueductos, teniendo en cuenta la rugosidad de las tuberías, línea del gradiente hidráulico y temperatura. Bogotá, Colombia. $1-154 \mathrm{p}$.

Haddad, A. 2019. Evaluation and correlation of friction head losses in smooth and rough pipes. Antalya, Turkey. Eurasia Proceedings of Science, Technology, Engineering \& Mathematics. 7(4):357-362.

Hassan, A. A. 2017. Design of sprinkler irrigation tapered main line by linear programming. Cayro, Egypt. Irrig. Drain 34(3):335-1350.

Jamil, R. 2019. Frictional head loss relation between Hazen-Williams and Darcy-Weisbach equations for various water supply pipe materials. Imam, Saudi Arabia. Int. J. Water. 13(4):333-347.

Jamil, R. and Mujeebu, M. A. 2019. Empirical relation between Hazen-Williams and DarcyWeisbach equations for cold and hot water flow in plastic pipes. Imam, Saudi Arabia. WATER. 108(6):104-114.

Jiménez-Jiménez, S. I. y Ramírez-Ruíz, C. 2018. Diseño hidráulico de tuberías con salidas múltiples mediante métodos numéricos. Aguascalientes, México. COMEII. 1-10 pp.

Melo, V. G. M. L.; Araújo, A. C. S.; Camargo, A. P.; Melo, L. L.; Frizzone, J. A. and Bombardelli, W. W. A. 2019. Head loss in thin-walled drip tapes with continuous labyrinth. Sao Paulo, Brazil. The Sci. World J. 2019(4)1-11.

Monge-Freile, M.; Sánchez-Delgado, M.; Huanca-Velarde, L. A. and Moreno-Llacza, A. 2019. Bamboo (Guadua angustifolia spp.) as a conduction alternative for a multi floodgates irrigation system. Lima, Perú. Anales Científicos. 80(1):240-252.

Monserrat, J.; Barragan, J. and Cots, L. 2018. Design of paired laterals on uniformly sloping fields. Lleida, Spain. J. Irrig. Drain. Eng. 144(6):1-14.

Nosrati, K.; Tahershamsi, A. and Taheri, S. H. S. 2017. Numerical analysis of energy loss coefficient in pipe contraction Using ANSYS CFX Software. Tehran, Iran. Civil Eng. J. 3(4):288-300.

Olivares, A.; Rodrigo, G. R.; Marco, A. M.; Notte, C. E. and Puentes, L. 2019. Experimental evaluation of correlations used to calculate friction factor for turbulent flow in cylindrical pipes. Serena. Revista Internacional de Métodos Numéricos para Cálculo y Diseño en Ingeniería. 35(1):1-6.

Pimenta, B. D.; Robaina, A. D.; Peiter, M. X.; Mezzomo, W.; Kirchner, J. H. and Ben, L. H. B. 2018. Performance of explicit approximations of the coefficient of head loss for pressurized conduits. Santa María, Brazil. Rev. Bras. Eng. Agr. Amb. 22(5):301-307. 
Sadeghi, S. H.; Peters, T. and Shelia, V. 2016. Energy grade line assessment for tapered microirrigation laterals. Washington, United States of America. J. Irrig. Drain. Eng. 142(7):1-11.

SARH. 1979. Secretaría de Agricultura y Recursos Hidraúlicos. Funcionamiento hidráulico, diseño y evaluación de sistemas de riego por goteo. Gómez Palacio, Durango, México. boletín 4 Centro Nacional de Métodos Avanzados de Riego (CENAMAR). 1-416 pp.

Taş, E. and Ağıralioğlu, N. 2018. Comparison of friction losses in long polyethylene pipe systems using different formulas. International Symposium on Urban Water and Wastewater Management Proceding. 602-609 pp.

Tas, E.; Agiralioglu, N.; Mehr, A. D. and Tur, R. 2020. Energy loss investigation in submarine pipelines: case study of cyprus water supply project. Antalya, turkey. Adv. Res. Civil Eng. 2(2):31-44.

Vilaça, F. N.; de Camargo, A. P.; Frizzone, J. A.; Mateos, L. and Koech, R. 2017. Minor losses in start connectors of microirrigation laterals. Sao Paulo, Brazil. Irrig. Sci. 35(3):227-240.

Wang, J. and Chen, R. 2019. An improved finite element model for the hydraulic analysis of drip irrigation subunits considering local emitter head loss. Jiangsu, China. Irrig. Sci. 38(17)1-16.

Wang, J.; Yang, T.; Wei, T.; Chen, R. and Yuan, S. 2019. Experimental determination of local head loss of non-coaxial emitters in thin-wall lay-flat polyethylene pipes. Jiangsu, China. Biosyst. Eng. 190(5):71-86.

Wang, J.; Zhu, D.; Bralts, V. F. and Zhang, L. 2016. Hydraulic analysis of looped microirrigation submain units using the finite element method. Yangling, China. American Society of Agricultural and Biological Engineers. 59(3):909-923.

Wang, Y.; Zhu, D.; Zhang, L. and Zhu, S. 2018. Simulation of local head los in trickle lateral lines equipped with in-line emitters based on dimensional analysis. Yichang, China. Irrigation and Drainage. Irrig. Sci. 67(4)1-10. 